\title{
Amiodarone Interactions with Membrane Lipids and with Growth of Bacillus stearothermophilus Used as a Model
}

\author{
Sónia M. L. J. Rosa, Maria C. Antunes-Madeira, \\ Amália S. Jurado, and Vítor V. M. C. Madeira* \\ Centro de Neurociências, Departamento de Zoologia, \\ Universidade de Coimbra, 3000 Coimbra, Portugal, \\ E-mail:vmcm@ci.uc.pt
}

Received December 1, 1999; Revised April 1, 2000; Accepted April 1, 2000

\begin{abstract}
The thermophilic eubacterium Bacillus stearothermophilus was used as a model to study the effects of amiodarone (2-butyl-3-[3',5'diido-4' $\alpha$-diethylaminoethoxybenzoyl]-benzofuran) in lipid organization and in bacterial growth. Effects on the structural order of lipids were assessed by fluorescence polarization of 1,6-diphenyl-1,3,5-hexatriene (DPH), probing the bilayer core, and of the propionic acid derivative 3-[p-(6-phenyl)-1,3,5hexatrienyl] phenylpropionic acid (DPH-PA), probing the outer regions of the bilayer. Amiodarone fluidizes bacterial polar lipid bilayers for temperatures below the phase transition midpoint, and orders the fluid phase of the bacterial polar lipids, as evaluated by DPH and DPH-PA. The ordering and disordering effects, which are concentration dependent, are more extensive when detected by DPH relative to DPH-PA. Growth studies performed in parallel revealed that amiodarone inhibits bacterial growth as a function of concentration. Amiodarone concentrations in the range from 1 to $2.5 \mu \mathrm{M}$ increased the lag time, decreased the specific growth rate, and decreased the final cell density. Furthermore, $3 \mu M$ amiodarone completely inhibited growth. These in vivo effects of amiodarone can be related to its ability to perturb the phospholipid bilayer structure, whose integrity is essential for cell function, viability, and growth.
\end{abstract}

Index Entries: Amiodarone; Bacillus stearothermophilus; fluorescent probes; membrane organization; bacterial growth.

*Author to whom all correspondence and reprint requests should be addressed. 